Trauma Berufskrankh 2007 - 9[Suppl 3]: S261-S263

DOI 10.1007/s10039-007-1281-9

Online publiziert: 21. September 2007

(c) Springer Medizin Verlag 2007
B. Förster

HVBG-Verwaltungsausschuss "Heilverfahren“,

Berufsgenossenschaft der Bauwirtschaft, Hauptverwaltung, Berlin

\section{Neuer Facharzt für das Durchgangsarzt- und Verletzungsartenverfahren}

\author{
Voraussetzungen - wer darf was?
}

Der Qualitätssicherung der medizinischen Rehabilitation in der gesetzlichen Unfallversicherung dienen im Bereich der Strukturqualität die besonderen Heilverfahrensarten wie

- das D- und das H-Arzt-Verfahren, durch die ein flächendeckendes Netz von niedergelassenen und im Krankenhaus tätigen qualifizierten Ärzten geschaffen wurde, und

- die Beteiligung von speziellen Kliniken am besonderen berufsgenossenschaftlichen Heilverfahren bei bestimmten Verletzungsarten (so genanntes Verletzungsartenverfahren).

In den folgenden Ausführungen werden die zurzeit zu erfüllenden Anforderungen für eine Beteiligung am Durchgangsarztund am Verletzungsartenverfahren dargestellt und die Gründe erläutert, die zu diesen Regelungen geführt haben. Um die gesamten direkten Beteiligungsmöglichkeiten des neuen Facharztes am berufsgenossenschaftlichen Heilverfahren darzustellen, wird auch kurz auf das H-ArztVerfahren eingegangen.

\section{Durchgangsarztverfahren}

Es ist neben dem Verletzungsartenverfahren wesentlicher Bestandteil der berufsgenossenschaftlichen Heilbehandlung. Der an die Träger der gesetzlichen Unfallversicherung gerichtete gesetzliche Auftrag einer rechtzeitigen und sachgemäßen besonderen unfallmedizinischen Rehabilitation wird über die Beteiligung hierfür ge- eigneter Mediziner und Einrichtungen realisiert.

Für die einzelnen Träger der gesetzlichen Unfallversicherung wählen die Landesverbände der gewerblichen Berufsgenossenschaften unter Beteiligung ihres jeweiligen beratenden Arztes und nach Besichtigung der Praxis die Durchgangsärzte aus. Die Beteiligung erfolgt über den Abschluss eines öffentlich-rechtlichen Vertrags.

Die Spitzenverbände der Träger der gesetzlichen Unfallversicherung haben die an die Durchgangsärzte gestellten Anforderungen für die Beteiligung einheitlich geregelt.

\section{Anforderungen}

Schon aufgrund des Beschlussantrags für den 104. Deutschen Ärztetag 2001 wurde die neue Weiterbildungsordnung frühzeitig in die Beratungen des Verwaltungsausschusses „Heilverfahren“ des Hauptverbands der gewerblichen Berufsgenossenschaften einbezogen. Ziel war es, den bisherigen hohen Standard des berufsgenossenschaftlichen Heilverfahrens zu erhalten und den Vorgaben der zu erwartenden neuen Weiterbildungsordnung gerecht zu werden. Zu Beginn der Diskussionen über die neue Weiterbildungsordnung war beabsichtigt, den Befähigungsnachweis für die Anerkennung der Facharztbezeichnung ausschließlich über Inhalte zu definieren. Weiterbildungszeiten vorzugeben, war zunächst grundsätzlich nicht vorgesehen; eine besondere unfall- chirurgische Zusatzqualifikation, vergleichbar mit dem Schwerpunkt „Unfallchirurgie“, ebenfalls nicht mehr.

Dies ließ uns einen Qualitätsverlust in der Versorgung Arbeitsunfallverletzter befürchten. Wir haben uns daher frühzeitig für eine Erhaltung sowohl einer qualitativ hochwertigen traumatologischen Versorgung Arbeitsunfallverletzter als auch einer unfallchirurgischen Weiterbildung im Sinne des bisherigen Schwerpunkts engagiert, denn eine Unterschreitung der bisherigen D-Arzt-Anforderungen, die ja den Schwerpunkt voraussetzten, würde die Qualität der Versorgung negativ beeinflussen. Daher konnte der neue Facharzt nicht automatisch zur Teilnahme am D-Arzt- und Verletzungsartenverfahren qualifizieren. Umso besser war es, dass unsere Vorstellungen sich mit der Beschlussfassung des 106. Deutschen Ärztetages 2003 zur neuen Musterweiterbildungsordnung deckten. Es war für uns daher nur konsequent, die entsprechende Regelung möglichst frühzeitig in die Anforderungen zur Beteiligung am berufsgenossenschaftlichen Heilverfahren zu integrieren, um den in der Aus- und Weiterbildung befindlichen Medizinern die Möglichkeit zu geben, sich auf die veränderten Rahmenbedingungen einstellen zu können. Die Unfallversicherungsträger konnten also davon absehen, eigene zeitliche und inhaltliche Vorgaben an eine unfallchirurgische Zusatzqualifikation zu definieren.

Als Ergebnis ist festzuhalten, dass nach den ab 01.01.2005 geltenden An- 
forderungen der Durchgangsarzt zum Führen der deutschen Facharztbezeichnung "Orthopädie und Unfallchirurgie“ berechtigt und als solcher niedergelassen oder an einem Krankenhaus oder einer Klinik fachlich und fachlich-organisatorisch weisungsfrei tätig sein muss. Er muss darüber hinaus über die deutsche Zusatzbezeichnung „Spezielle Unfallchirurgie" verfügen.

Für den Übergang wurden die bisherigen Anforderungen an die fachliche $\mathrm{Be}$ fähigung beibehalten.

Aktuell sind in Deutschland insgesamt 3428 Durchgangsärzte an der berufsgenossenschaftlichen Heilbehandlung beteiligt.

\section{Aufgaben}

Bei der Erstvorstellung hat der Durchgangsarzt die Aufgabe:

- den Verletzten eingehend zu untersuchen,

- die Diagnose zu stellen und

- die Verletzung fachgerecht ärztlich zu versorgen.

In dem dem Unfallversicherungsträger zu erstattenden Durchgangsarztbericht hat der Durchgangsarzt auch zur Kausalität zwischen geschildertem Unfallhergang und Verletzung Stellung zu beziehen.

Aufgrund des erhobenen Befunds entscheidet der Durchgangsarzt für den Unfallversicherungsträger,

- ob eine allgemeine Behandlung durch den Hausarzt ausreichend ist oder

- ob der Verletzte in seiner besonderen Heilbehandlung verbleiben sollte.

Letztere erscheint dann angezeigt, wenn durch sie eine Verkürzung der Behandlungsdauer oder der Arbeitsunfähigkeit oder eine bessere Wiederherstellung des Verletzten zu erwarten ist. Im Falle der allgemeinen Behandlung erfolgt die primäre Therapie durch den Allgemeinmediziner bzw. Hausarzt. Das Heilverfahren wird durch Nachschauberichte des D-Arztes überprüft. Hierbei hat dieser den Verletzten zu untersuchen und erneut $\mathrm{zu}$ entscheiden, ob die allgemeine Behandlung weiterhin ausreichend ist oder ob jetzt besondere Behandlung notwendig wird.

\section{H-Arzt-Verfahren}

Der wesentliche Unterschied des H-Arztzum D-Arzt-Verfahren besteht in der fehlenden Entscheidungsmöglichkeit des $\mathrm{H}$ Arztes hinsichtlich der Art der einzuleitenden Behandlung. Er ist nur bei bestimmten, in einem Katalog definierten Verletzungen berechtigt, besondere Heilbehandlung einzuleiten. Eine Vorstellungspflicht an den Durchgangsarzt besteht aber nicht.

Über den Antrag auf Beteiligung als H-Arzt entscheiden - anders als beim Durchgangsarzt - nicht die Unfallversicherungsträger allein. Entsprechend dem Vertrag Ärzte/Unfallversicherungsträger bestimmt über die Beteiligung ein Ausschuss, der sich aus je 3 Vertretern der zuständigen Kassenärztlichen Vereinigung und des zuständigen Landesverbands der gewerblichen Berufsgenossenschaften zusammensetzt.

Ab 01.01.2005 wird im berufsgenossenschaftlichen Heilverfahren der besseren Qualifikation des neuen Facharztes dadurch Rechnung getragen, dass dieser nun unmittelbar als $\mathrm{H}$-Arzt beteiligt werden kann. Bis Ende 2004 musste zusätzlich eine mindestens 2-jährige unfallmedizinische Tätigkeit in einer mit einem D-Arzt besetzten Krankenhausabteilung nachgewiesen werden. Für den Übergang wurden auch hier die bisherigen Anforderungen an die fachliche Befähigung beibehalten. $\mathrm{Ab}$

01.01.2006 sehen die Anforderungen nur die Beteiligung niedergelassener Ärzte vor.

Zurzeit sind 3084 H-Ärzte an der berufsgenossenschaftlichen Heilbehandlung beteiligt.

\section{Verletzungsartenverfahren}

Unfallverletzte mit bestimmten schweren Verletzungen bedürfen unmittelbar nach dem Unfall einer besonderen stationären Behandlung. Das zu diesem Zweck eingeführte Verletzungsartenverfahren regelt die Einweisung in eine besondere fachärztliche stationäre Behandlung. Werden die gestellten Anforderungen erfüllt, kann ein Krankenhaus von den Berufsgenossenschaften an der Behandlung entsprechender Verletzungen beteiligt werden.
Die in einem besonderen Katalog aufgeführten Verletzungen dürfen nur in einem solchen, von den Berufsgenossenschaften beteiligten Krankenhaus stationär behandelt werden.

\section{Anforderungen}

Das entsprechende Krankenhaus muss über die modernsten Einrichtungen zur Behandlung von Unfallverletzten verfügen.

Der Chefarzt bzw. leitende Arzt muss neben einer Durchgangsarztbeteiligung besondere aktuelle unfallmedizinische Erfahrungen haben. Die Anforderungen gehen hierzu von einer mindestens 3jährigen Tätigkeit in einem am Verletzungsverfahren beteiligten Krankenhaus aus. Daneben muss er über die Weiterbildungsbefugnis im Schwerpunkt „Unfallchirurgie" oder die Zusatzweiterbildung „Spezielle Unfallchirurgie“ verfügen, die im Regelfall 2 Jahre nicht unterschreiten soll.

\section{Problematik Landesärztekammer}

Leider müssen wir feststellen, dass die Umschreibung des bisherigen Schwerpunkts „Unfallchirurgie“ auf die Zusatzbezeichnung „Spezielle Unfallchirurgie“ von den einzelnen Landesärztekammern sehr unterschiedlich gehandhabt wird. Werden in einigen Kammern die Inhaber der bisherigen Schwerpunktbezeichnung „Unfallchirurgie“ automatisch überführt, müssen diese in anderen Kammern eine Prüfung ablegen.

In allen Landesärztekammern ist vor der Erteilung einer Weiterbildungsbefugnis für die Zusatzbezeichnung „Spezielle Unfallchirurgie“ ein Antrag erforderlich. Eine automatische Übernahme der bisherigen Weiterbildungsbefugnis erfolgt somit nicht. Weil die Anträge von den Landesärztekammern anschließend auch unter Berücksichtigung der aktuellen Verhältnisse der Weiterbildungsstätte geprüft werden, kann es durchaus zu einer Ablehnung der bisher bestehenden Weiterbildungsbefugnis oder zu einer Verkürzung der Weiterbildungsdauer kommen.

Die weitere Entwicklung in diesem Zusammenhang wird von den Berufsgenossenschaften gezielt beobachten wer- 
den, damit diese unterschiedlichen Verfahrensweisen in den Landesärztekammern nicht zu Lasten der Versorgung der Schwerverletzten führen.

\section{Aktuelle Situation}

Am Verletzungsartenverfahren sind zurzeit 606 Krankenhäuser beteiligt.

\section{Resümee}

Bei obigen Ausführungen, wie sich die Träger der gesetzlichen Unfallversicherung den Bedingungen der neuen Weiterbildungsordnung gestellt haben, handelt es sich natürlich um eine Momentaufnahme. Die Berufsgenossenschaften sind sich bewusst, dass die unterschiedlichen Inhalte und Anforderungen an den „Schwerpunkt Unfallchirurgie“ einerseits und die Zusatzweiterbildung „Spezielle Unfallchirurgie“ andererseits evtl. Quantitätsprobleme bei der Versorgung der Unfallverletzten mit sich bringen können. Bei einer solchen sich abzeichnenden Entwicklung werden sie umgehend reagieren.

Nach wie vor gilt laut Reformpapier zur gesetzlichen Unfallversicherung (Eckpunkte der Staatssekretäre vom 29.06.2006):

„Die Heilbehandlung wird weiterhin mit allen geeigneten Mitteln erbracht. Damit wird der hohe qualitative Standard in der Unfallversicherung auch in Zukunft gewährleistet..."

\section{Korrespondenzadresse \\ B. Förster}

HVBG-Verwaltungsausschuss "Heilverfahren“, Berufsgenossenschaft der Bauwirtschaft, Hauptverwaltung,

Hildegardstraße 29/30, 10715 Berlin

bernhard.foerster@bgbau.de

Interessenkonflikt. Keine Angaben

Trauma Berufskrankh 2007 · 9[Suppl 3]: S261-S263 DOI 10.1007/s10039-007-1281-9

(c) Springer Medizin Verlag 2007

\section{B. Förster \\ Neuer Facharzt für das Durchgangsarzt- und Verletzungs- artenverfahren. Voraussetzungen - wer darf was?}

\section{Zusammenfassung}

Die zurzeit zu erfüllenden Anforderungen für eine Beteiligung am Durchgangsarzt- (DArzt-) und am Verletzungsartenverfahren werden dargestellt und die Gründe erläutert, die zu diesen Regelungen geführt haben. Zur Teilnahme am D-Arzt-Verfahren ist nach wie vor eine besondere Qualifikation erforderlich, denn die Durchgangsärzte sind für die Umsetzung des an die Träger der gesetzlichen Unfallversicherung gerichteten gesetzlichen Auftrags einer rechtzeitigen und sachgemäßen besonderen unfallmedizinischen $\mathrm{Re}$ habilitation maßgeblich mitverantwortlich. Sie entscheiden - im Gegensatz zum H-Arzt über die Art der einzuleitenden Behandlung. Auch die am Verletzungsartenverfahren be-

\section{A new specialist for emergency and injury typing procedures. Conditions - who is allowed to do what?}

\section{Abstract}

The requirements that currently have to be met to allow involvement in emergency and injury typing procedures are introduced, and the reasons that have led to them are explained. As always, a special qualification is still needed before a doctor can take part in emergency procedures, as the emergency call-out doctors bear a decisive share of the responsibility for implementation of the statutory tasks of prompt and appropriate specific traumatological rehabilitation that devolve on the statutory accident insurance scheme. In contrast to the doctors accepted as qualified to apply treatments approved by the employers' liability insurance associations, the emergency doctors decide on the kind of treatment to be initiated. The hospi- teiligten Krankenhäuser müssen besondere stationäre und personelle Anforderungen erfüllen. Auf die derzeit unterschiedliche Handhabung der Umschreibung des bisherigen Schwerpunkts „Unfallchirurgie" auf die Zusatzbezeichnung „Spezielle Unfallchirurgie“ durch die Landesärztekammern wird von den Berufsgenossenschaften besonders geachtet, um bei Problemen rechtzeitig einschreiten zu können.

\section{Schlüsselwörter}

Durchgangsarztverfahren · Verletzungsartenverfahren · Qualifikation · Schwerpunktumschreibung · Landesärztekammer tals in which the injury typing procedure is applied are also required to fulfil specific requirements concerning patient accommodation and staff. The insurance associations pay particular attention to the currently variable management of how the medical councils of the länder redefine the emphasis of trauma surgery used hitherto fit the supplementary designation of special trauma surgery, so as to be able to step in promptly in the event of any problems.

\section{Keywords}

Emergency medical procedures · Injury typing procedures - Qualifications . Redefinition of emphasis · Medical councils 